\title{
Study of Sensing Hazardous Insecticides - Molecule Adsorption on Pristine Single-Walled Carbon Nanotubes
}

\author{
Saja H. Kareem*, Falah H. Hanoon \\ Department of Physics, Faculty of Science, University of Thi Qar, Nassiriya 64001, Iraq.
}

\section{ARTICLE DETAILS}

Article history:

Received 26 August 2019

Accepted 06 September 2019

Available online 24 September 2019

\section{Keywords:}

Insecticide

Carbon Nanotube

DFT Study

\begin{abstract}
A B S T R A C T
A computational study, density functional theory (DFT) have been applied on the adsorption of DDD, DDE and DDT molecules on $(8,0)$ pristine single-walled carbon nanotubes (PSWCNTs). The DDD, DDE and DDT molecules adsorption are aggressively favorable with adsorption energy $\left(E_{a d s}\right)$ equal to -67.266 , -58.776 and $-52.245 \mathrm{eV}$ respectively. In this study, the electronic properties analysis reveals that the physisorbed DDD, DDE and DDT are changing the highest occupied molecular orbital (HOMO) energy gap and lowest unoccupied molecular orbital (LUMO) energy gap of carbon nanotubes which have been reduced after interaction with these molecules from $0.519 \mathrm{eV}$ to $0.356,0.289$ and $0.296 \mathrm{eV}$ respectively. These three insecticide molecules make changes outside of the carbon nanotube to the set of energy molecule and thus nanotubes are obtained as conductive structure. As inference it reveals that the pristine single-walled carbon nanotubes can be a good sensor for the detection of DDD, DDE and DDT molecules.
\end{abstract}

\section{Introduction}

Since the discovery of carbon nanotubes CNTs in 1991, it has been intrigued broad interdisciplinary courtesies. CNTs and nanoparticles with low dimension and high aspect ratio surface-to-volume ratio are presenting unique physical, chemical, mechanical and permanent electrical properties [1-3]. CNTs and nanoparticles have a wide range of important applications including nanoelectric devices, energy storage, and catalyst [4-6].

They can detect even a small concentration of the analyze, they can be utilized in chemical and biological sensors [1-7]. CNTs can be either single-walled (SW) with discrete fragment of curved carbon sheet, or multi-walled (MW). They can be either metallic or semiconducting dependent on their chirality defined with a pair of integer indices (n, m) [8]. CNTs can be classified to armchair (n, n), zig-zag, (n, 0) and chiral $(\mathrm{n}, \mathrm{m})$. The armchair nanotubes always show a metallic behavior with high current capacity and the ballistic nature of electron transport. They always maintain metallic property and mechanic stability under the compressive and tensile strains. Thus, the current variation in the armchair nanotube is more stable under small uniaxial strains, and this characteristic could be applied to thermal strain [9].

So armchair SWCNT based chemical sensors are effective devices to detect various molecules. Fast response time and high sensitivity at room temperature are the most important characteristics of these sensors to detect contaminant molecules [10]. SWCNTs cannot detect some gaseous molecule such as carbon monoxide or ammonia because of the weak Van der Waals interaction and insufficient charge transfer between SWCNT surface and molecules. Limited environmental sensitivity of pristine CNTs which results from smooth surface low adsorption energy, affinity and long recovery time could be modified by functionalization. Chemical modification is an effective approach to overcome this restriction and improve the binding strength by the enhancement of the chemical reactivity of SWCNTs. Although the electronic properties of CNTs are strongly depends on the delocalized p-electron conjugation system that make the CNTs electrochemically active [11].

Use of insecticides in agriculture is a common practice over the whole world. These insecticides or pesticides help to increase agricultural productivity by killing insects selectively. However, they can have some serious hazardous effect on human body as well. One such widely used

*Corresponding Author:msc.saja_phy@sci.utq.edu.iq(Saja H. Kareem) insecticide is dichlorodiphenyltrichloroethane (DDT). Because of its adverse effect on environment [12-14], use of this pesticide has been banned in many countries. But DDT has been still found to be contaminating agricultural soil and water in many places. It further exhibits very slow degradation rate, which can take upto 30 years. By getting accumulated in plants and animals, DDT can further show bio magnification effect as we go up in a food chain [15]. Its metabolites dichlorodiphenyldichloroethane (DDD) and dichlorodiphenyldichloro ethylene (DDE) and both are equally harmful to human body [16].

\section{Experimental Methods}

\subsection{Computational Methodology}

In this work, the adsorption behavior of the DDD, DDE and DDT molecule were considered on zigzag single wall $(8,0)$ CNT. All the optimizations and energy calculations were performed using Gaussian 09 program package at the level of density functional theory (DFT) with B3LYP/6-31G. The binding energies ( $\mathrm{E}_{\text {ad }}$ ) of DDD, DDE and DDT on the CNT are determined through the following equation,

$$
\mathrm{E}_{a d}=E_{C N T \text {-molecule }}-\left(E_{C N T}+E_{\text {molecule }}\right)
$$

where $E_{C N T-\text { molecule }}$ is the total energy for the interaction with DDD, DDE and DDT. $E_{C N T}$ is the total energy of the CNT and $E_{\text {molecule }}$ is the total energy of an isolated DDD, DDE and DDT molecule.

\section{Results and Discussion}

\subsection{Adsorption of DDD on CNT}

This work is investigating the adsorption of DDD, DDE and DDT on the exterior surface of the CNT. Confinement of the DDD, DDE and DDT are in outside of $(8,0)$ carbon nanotube at distance $12 \AA$. All confined DDD, DDE and DDT molecules outside CNT (Fig. 1) were optimized with DFT method. Interestingly after full relaxation with no constraints, exothermic adsorption energies ( $E_{\text {ads }}$ ) were obtained as $-67.266,-58.776$ and -52.245 $\mathrm{eV}$ respectively.

DDD, DDE and DDT were adsorbed externally on the carbon nanotube (Fig. 1). The DFT calculations show that the adsorption of DDD, DDE and DDT molecule on the carbon atoms are an exothermic process with negative $E_{\text {ads }}-67.266,-58.776$ and $-52.245 \mathrm{eV}$ and interaction distance of $2.777,2.777$ and $2.224 \AA$ and hence these interactions indicating that the 
DDD, DDE and DDT are physisorbed on the CNT. Table 1 indicating that the DDD acts as an electron acceptor and the CNT as an electron donor. Performance of a DFT analysis on the DDD, DDE and DDT molecules are showing that its HOMO is slightly more localized. Therefore, the interaction between the molecules and $\mathrm{C}_{\mathrm{Lumo}}$ of nanotube is localized on $\mathrm{C}$ atom as somewhat strong.

Table 1 The highest occupied molecular orbital (HOMO) energy ( $\mathrm{E}_{\text {номо }}$ ), the lowest unoccupied molecular orbitals (LUMO) energy (ELUMo), the adsorption energies Eads, the HOMO-LUMO gap $\left(\mathrm{E}_{\mathrm{g}}\right)$ total energy and sensitivity of mentioned systems

\begin{tabular}{lllllll}
\hline System & Eномо $_{\text {HeV }}$ & E Lumo $_{(\mathrm{eV})}$ & $\mathrm{E}_{\mathrm{g}}(\mathrm{eV})$ & $\mathrm{E}_{\text {ads }}(\mathrm{eV})$ & $\mathrm{E}_{\text {total }}(\mathrm{eV})$ & Sensitivity \\
\hline CNT & -6.089 & -5.570 & 0.519 & - & -98927 & - \\
CNT-DDD & -5.248 & -5.892 & 0.356 & -67.266 & -163296 & 0.314 \\
CNT-DDE & -5.492 & -5.203 & 0.289 & -58.776 & -163277 & 0.443 \\
CNT-DDT & -5.224 & -4.928 & 0.296 & -52.245 & -175731 & 0.429 \\
\hline
\end{tabular}

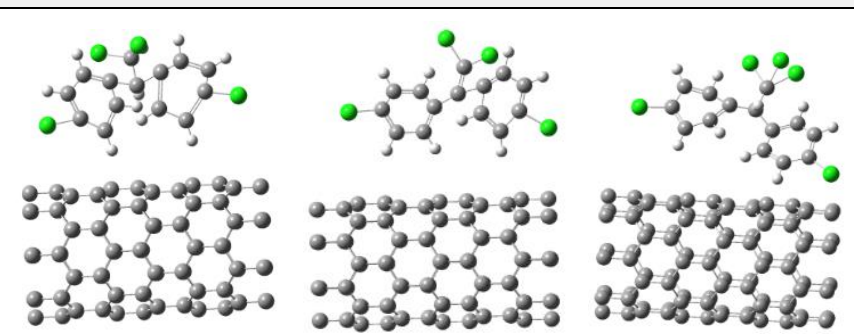

Fig. 1 The structural geometric of (A) CNT-DDD, (B) CNT-DDE and (C) CNT-DDT

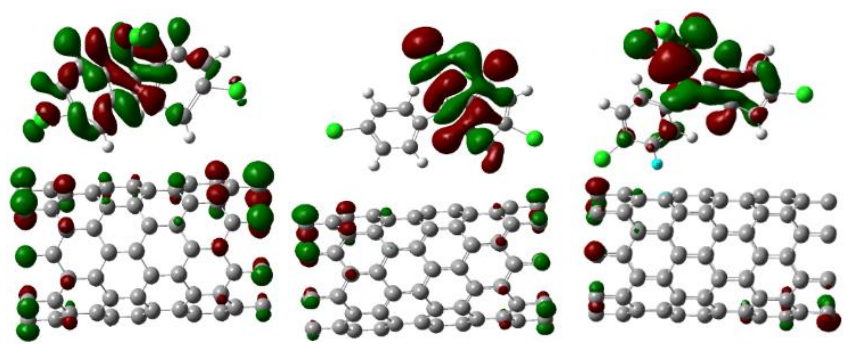

Fig. 2 HOMO-LUMO electron density for all systems

\subsection{The DOS of the (Molecule/Nanotube) Systems}

To validate the effects of DDD, DDE and DDT molecules adsorption on the nanotube, electronic properties, electronic density of states (DOS) of the nanotube and adsorbate/nanotube complexes were calculated (Fig. 3). For all three configurations, it can be found that DOS are not affected by the DDD, DDE and DDT adsorption (Fig. 3). So, the $\mathrm{E}_{\mathrm{g}}$ of the nanotube has no significant change externally (DDD adsorptions $\mathrm{Eg}=0.356 \mathrm{eV}$, DDE adsorptions $\mathrm{Eg}=0.289 \mathrm{eV}$ and DDT adsorptions $\mathrm{Eg}=0.296 \mathrm{eV}$ ). This occurrence is expected to bring about obvious change in the corresponding electrical conductivity because it is well known that the energy gap or band gap in bulk materials is a major factor determining the electrical conductivity of a material and a classic relation between them is as follows:

$$
\sigma \alpha \exp \left(\frac{-E g}{2 K T}\right)
$$

where $\sigma$ is the electrical conductivity and k is the Boltzmann's constant.

According to Eq.(2), the smaller $\mathrm{E}_{\mathrm{g}}$ at a given temperature leads to the higher electrical conductivity. However, the energy gap of DDD, DDE and DDT/nanotube complex are quantitatively reduced compared to that of the nanotube. Since the conductivity is exponentially correlated with negative value of $E_{g}$, it is expected that it become larger with reducing the energy gap. It demonstrates the high sensitivity of the electronic properties of the carbon nanotube towards the adsorption of the DDD, DDE and DDT on molecules. It is believed that the carbon nanotube can transform the presence of the molecules directly into an electrical signal.
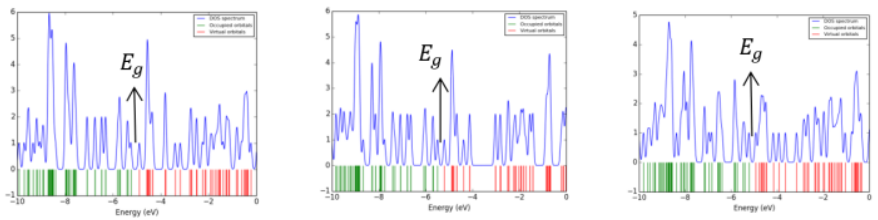

Fig. 3 Calculated density of states DOS for a) CNT-DDD, b) CNT-DDE and c) CNT-DDT

\section{Conclusion}

This work has been performed a computational study of density functional theory (DFT) calculations to study the electronic properties of DDD, DDE and DDT molecules which are adsorbed in the surface of pristine carbon nanotube. It was found that the three molecules can be strongly adsorbed in the nanotube exterior surface with high adsorption energies, and changes energy gap which leads to increase the electrical conductivity. Charge transfer between the molecule and CNTs generates the sensing property of the CNTs. Importantly, it was deduced that the pristine carbon nanotube can acts as a good sensor for DDD, DDE and DDT molecules.

\section{References}

[1] Z. Wang, M. Devel, B. Dulmet, S. Stuart, Geometry-dependent nonlinear decrease of the effective Young's modulus of single-walled carbon nanotubes submitted to large tensile loadings, Fullerenes Nanotubes Carbon Nanostruct. 17 (2009) 1-10.

[2] T. Zhang, S. Mubeen, N.V. Myung, M.A. Deshusses, Recent progress in carbon nanotube-based gas sensors, Nanotechnol. 19 (2008) 332001:1-14.

[3] B.J. Sanghavi, W. Varhue, A. Rohani, K.T. Liao, L.A. Bazydlo, et al., Ultrafast immunoassays by coupling dielectrophoretic biomarker enrichment in nanoslit channel with electrochemical detection on graphene, Lab Chip. 15 (2015) 4563-4570.

[4] M. Yoosefian, H. Raissi, A. Mola, The hybrid of Pd and SWCNT (Pd loaded on SWCNT) as an efficient sensor for the formaldehyde molecule detection: a DFT study, Sens. Actuators B Chem. 212 (2015) 55-62.

[5] M. Yoosefian, M. Zahedi, A. Mola, S. Naserian, A DFT comparative study of single and double $\mathrm{SO}_{2}$ adsorption on Pt-doped and Au-doped single-walled carbon nanotube, Appl. Surf. Sci. 349 (2015) 864-869.

[6] M. Yoosefian, N. Etminan, Pd-doped single-walled carbon nanotube as a nanobiosensor for histidine amino acid, A DFT study, RSC Adv. 5 (2015) 31172-31178.

[7] R.N. Goyal, V.K. Gupta, N. Bachheti, Fullerene-C60-modified electrode as a sensitive voltammetric sensor for detection of nandrolone-an anabolic steroid used in doping, Anal. Chim. Acta 597 (2007) 82-89.

[8] R. Saito, M. Fujita, G. Dresselhaus, U.M. Dresselhaus, Electronic structure of chiral graphene tubules, Appl. Phys. Lett. 60 (1992) 2204-2206.

[9] Y.R. Chen, C.I. Weng, S.J. Sun, Electronic properties of zigzag and armchair carbon nanotubes under uniaxial strain, J. Appl. Phys. 104 (2008) 114310:1-8

[10] S. Peng, K. Cho, Ab initio study of doped carbon nanotube sensors, Nano Lett. 3 (2003) 513-517.

[11] L. Duclaux, Review of the doping of carbon nanotubes (multiwalled and singlewalled), Carbon 40 (2002) 1751-1764.

[12] K.H. Kim, E. Kabir, S.A. Jahan, Exposure to pesticides and the associated human health effects, Sci. Total Environ. 575 (2017) 525-535.

[13] I.C. Yadav, N.L. Devi, J.H. Syed, Z. Cheng, J. Li, et al., Current status of persistent organic pesticides residues in air, water, and soil, and their possible effect on neighboring countries: A comprehensive review of India, Sci. Total Environ. 511 (2015) 123-137.

[14] M. Van Den Berg, K. Kypke, A. Kotz, A. Tritscher, S.Y. Lee, et al., WHO/UNEP global surveys of PCDDs, PCDFs, PCBs and DDTs in human milk and benefitrisk evaluation of breastfeeding, Arch. Toxicol. 91 (2017) 83-96.

[15] S. Bayen, P. Giusti, H.K. Lee, P.J. Barlow, P.J. Obard, WHO/UNEP global surveys of PCDDs, PCDFs, PCBs and DDTs in human milk and benefit-risk evaluation of breastfeeding, J. Toxi. Environ. Heal. A 68 (2005) 51-65.

[16] J.A. Bumpus, S.D. Aust, Biodegradation of DDT [1,1,1-trichloro-2,2-bis(4 chlorophenyl)ethane] by the white rot fungus Phanerochaete chrysosporium, Appl. Environ. Microbiol. 53 (1987) 2001-2008. 